University of Wollongong

Research Online

Faculty of Engineering and Information

Faculty of Engineering and Information

Sciences - Papers: Part A

Sciences

$1-1-2013$

Description and comparison of documentation of nursing assessment between paper-based and electronic systems in Australian aged care homes

Ning Wang

University of Wollongong, nw57@uowmail.edu.au

Ping Yu

University of Wollongong, ping@uow.edu.au

David Hailey

University of Wollongong, dhailey@uow.edu.au

Follow this and additional works at: https://ro.uow.edu.au/eispapers

Part of the Engineering Commons, and the Science and Technology Studies Commons

Research Online is the open access institutional repository for the University of Wollongong. For further information contact the UOW Library: research-pubs@uow.edu.au 


\title{
Description and comparison of documentation of nursing assessment between paper-based and electronic systems in Australian aged care homes
}

\begin{abstract}
Purpose: To describe nursing assessment documentation practices in aged care organizations and to evaluate the quality of electronic versus paper-based documentation of nursing assessment. Methods: This was a retrospective nursing documentation audit study. Study samples were 2299 paper-based and 6997 electronic resident assessment forms contained in 159 paper-based and 249 electronic resident nursing records, respectively, from three aged care organizations. The practice of nursing assessment documentation in participating aged care homes was described. Three attributes of quality of nursing assessment documentation were evaluated: format and structure, process, and content by seven measures: quantity, completeness, timeliness comprehensiveness, frequencies of documentation specific to care domains and data items, and whether assessment forms were signed and dated. Results: Varying practice in documentation of nursing assessment was found among different aged care organizations and homes. Electronic resident records contained higher numbers and more comprehensive resident assessment forms than paper-based records. The frequency of documentation was higher in electronic than in paper-based records in relation to most care domains. There was no difference between the two types of documentation systems on other aspects of nursing assessment documentation (overall completeness and timeliness, variation of frequencies among different care domains, and item completion in personal hygiene assessment forms). Conclusions: Electronic nursing documentation systems could improve the quality of documentation structure and format, process and content in the aspects of quantity, comprehensiveness and signing and dating of assessment forms. Further studies are needed to understand the factors leading to the variations of practice and the limitations of nursing assessment documentation and to evaluate documentation quality from a clinical perspective. (c) 2013 Elsevier Ireland Ltd.
\end{abstract}

\section{Keywords}

sElectronic health records, Paper records, Assessment forms, Audit, Evaluation, Quality, Aged care

Disciplines

Engineering | Science and Technology Studies

\section{Publication Details}

Wang, N., Yu, P. \& Hailey, D. (2013). Description and comparison of documentation of nursing assessment between paper-based and electronic systems in Australian aged care homes. International Journal of Medical Informatics, 82 (9), 789-797. 


\section{Title page}

Title of the manuscript: Description and comparison of documentation of nursing assessment between paper-based and electronic systems in Australian aged care homes

\section{Authors of the manuscript:}

Ning Wang, RN, PhD, MPH

Ping Yu, PhD

David Hailey, PhD

Affiliation: Health Informatics Research Laboratory, School of Information Systems and Technology, Faculty of Informatics, University of Wollongong

The corresponding author: Ping Yu: PhD Senior Lecturer

Health Informatics Research Laboratory, School of Information System and Technology, Faculty of Informatics, University of Wollongong, Northfields Ave, Wollongong NSW 2522, Australia

Email: ping@uow.edu.au

Phone: +612 42215412

Fax: +612 42214045

Website: http://www.uow.edu.au/ ping/

Key words: nursing documentation, electronic health records, EHR, paper records, assessment forms, audit, evaluation, quality, aged care, long-term care. 


\begin{abstract}
Purpose: To describe nursing assessment documentation practices in aged care organizations and to evaluate the quality of electronic versus paper-based documentation of nursing assessment.
\end{abstract}

Methods: This was a retrospective nursing documentation audit study. Study samples were 2299 paper-based and 6997 electronic resident assessment forms contained in 159 paper-based and 249 electronic resident nursing records, respectively, from three aged care organizations. The practice of nursing assessment documentation in participating aged care homes was described. Three attributes of quality of nursing assessment documentation were evaluated: format and structure, process, and content by seven measures: quantity, completeness, timeliness comprehensiveness, frequencies of documentation specific to care domains and data items, and whether assessment forms were signed and dated.

Results: Varying practice in documentation of nursing assessment was found among different aged care organisations and homes. Electronic resident records contained higher numbers and more comprehensive resident assessment forms than paper-based records. The frequency of documentation was higher in electronic than in paper-based records in relation to most care domains. There was no difference between the two types of documentation systems on other aspects of nursing assessment documentation (overall completeness and timeliness, variation of frequencies among different care domains, and item completion in personal hygiene assessment forms). Conclusions: Electronic nursing documentation systems could improve the quality of documentation structure and format, process and content in the aspects of quantity, comprehensiveness and signing and dating of assessment forms. Further studies are needed to understand the factors leading to the variations of practice and the limitations of nursing assessment documentation and to evaluate documentation quality from a clinical perspective. 


\section{Introduction}

Electronic nursing documentation systems, which aggregate digital forms of data about clients and nursing care, have been increasingly introduced into care settings to assist nurses’ daily work [1]. Such systems are able to improve documentation quality and allow reuse of data for various purposes, albeit with mixed overall effects on nursing care [2, 3, 4].

Given the potential of information and communication technology (ICT) in supporting care provision, several aged care organisations have implemented electronic nursing documentation systems. These systems were expected to improve the quality of documentation and communication among care team members to facilitate care, and to provide better evidence that care services meet accreditation standards and government requirements.

Nursing documentation, a major clinical information source in Australian aged care, plays a vital role in the delivery of aged care services [5]. Resident assessment forms recording information about nursing assessment are a significant part of a resident's record. Nursing assessment is the first stage of the nursing process and an important task in daily care practice. During assessment, a nurse systematically collects, verifies, analyses and communicates a health care client's information to derive a nursing diagnosis and to plan individualized care for the client [6]. The accuracy and completeness of nursing assessment determines the accuracy of follow-on other steps of the nursing process [7].

In aged care, nursing assessment documentation practice is shaped by the aged care accreditation standards in the sense that the scope of care to be assessed has been defined. This scope includes clients’ care issues from health, personal care and safety 
to a range of lifestyle matters [8]. However, it is unclear how nursing assessment documentation is conducted at an operational level in individual aged care organisations or nursing homes.

Previous studies have addressed the content of nursing assessment documentation at different levels, considering whether and/or to what extent general or specific assessment data have been documented. For example, Björvell et al. [9] and Darmer et al. [10] measured the presence of the 19 pre-structured nursing status keywords in the VIPS documentation system such as communication, nutrition and pain on a patient's arrival and ongoing updates of care needs. At a specific care level, Barry et al. [11] identified poor documentation of vital signs and physical assessment for residents with acute change in condition in nursing home settings. Ehrenberg et al. [12] assessed the presence of relevant assessment data in relation to chronic heart failure.

Measurement approaches used in previous studies to only address the documentation content appear to be inadequate to comprehensively reflect the capacity of electronic documentation systems to assist nurses to undertake and document nursing assessment. In fact, the quality of documentation has three attributes: structure and format, process, and content, which refer to data presentation, collection and meaning [13].

To obtain an understanding about nursing documentation practice relating assessment and to describe the effect of the electronic systems on the quality of nursing documentation, a nursing documentation audit on nursing assessment forms was carried out. The aims of the study were to investigate nursing assessment documentation practice in Australian aged care homes and to compare the quality of 
nursing assessment documentation in the paper-based and electronic nursing systems. For this study, multiple measures were developed to address the three quality attributes of assessment documentation.

\section{Method}

\section{1 Study design}

This was a nursing documentation audit study. A retrospective review of paper-based and electronic resident assessment forms was carried out.

\section{2 Study sites}

The study was conducted at nine residential aged care homes belonging to three aged care organisations in Australia: Organisation 1 (Homes A and B), Organisation 2 (Homes C, D and E) and Organisation 3 (Homes F, G, H and I). Electronic documentation systems had been implemented in the aged care homes at different times since 2005. The documentation audit was carried out between August 2010 and June 2011.

\section{3 Participants}

The study participants were residents whose nursing assessment forms were accessed by the researchers with the informed consent of the residents and/or their families. The residents were considered eligible for the study if they had stayed in the nursing home long enough to undergo a complete admission assessment.

\section{4 Study sample}

The study samples were resident assessment forms contained in a total of 159 paperbased and 249 electronic resident records. These records were selected using 
convenience sampling. The sample size was related to the number of resident records or number of particular assessment forms as determined by the nature of measurement items. It could vary among measurement items, depending on the availability of records or assessment forms.

\section{5 Measurement approaches}

Seven criteria were developed to measure and compare the quality attributes of structure and format, process and content of nursing assessment documentation in paper-based and electronic systems:

The quantity of nursing assessment documentation (format and structure): two methods were used: the percentage of resident records containing nursing assessment forms and the mean number of assessment forms in a record.

Completeness of resident admission and ongoing assessment forms (format and structure): this was defined as the degree to which items in an assessment form were filled in by a nurse. A five-point Likert scale from 0 to 4 was used to grade each form according to the proportion of items in the form that had been completed. Scores given were 4 for $100 \%$ of items completed, 3 for $66.6 \%$ to $100 \%$, 2 for $33.3 \%$ to $66.6 \%$ and 1 for less than $33.3 \%$. A score of zero was given to a form if all items were incomplete. As each resident's record may have a number of assessment forms, the mean score of assessment forms was used for each record.

Timeliness of nursing assessment documentation (process): this measured the extent to which admission assessment forms were documented within a timeframe required by the relevant aged care organisation. The scoring system for completeness assessment shown above was used for this measure. For example, a score of 3 was 
given to a record if between $66.6 \%$ and $100 \%$ of admission assessment forms were completed as required by the organisation within 28 days of the resident's admission.

Comprehensiveness of nursing assessment documentation (content): this referred to the extent to which documented assessment forms covered the required scope of care needs. For this measure, a checklist with a range of assessment domains was established in accordance with the aged care accreditation standards and the existing aged care documentation practice in the participating organisations. It contains 16 domains (see Figure I) that were validated with five nursing managers to reaching a full consensus. The comprehensiveness rate was calculated using the following formula:

$$
\begin{gathered}
\text { Comprehensiveness rate }=(\text { Number of assessment domains with presentation of } \\
\text { documentation/Total number of domains }) \times 100
\end{gathered}
$$

Frequency of documentation of assessment data specific to a care domain (content): this measured the extent to which resident care needs specific to the care domains on the checklist such as pain and mobility were assessed and recorded by the nursing staff. The following formula was used to calculate the frequency proportion:

Frequency proportion $=$ (Number of records with documentation specific to a care domain/ Total number of records audited) x 100

The frequency of documentation of data specific to each item in an assessment form (content): this measure addressed the extent of documentation at each item level to reflect how much specific data was collected to indicate a resident's care needs. The resident personal hygiene assessment forms were used as a case study. A formula for calculating this measure is: 
Frequency of documentation of data item $=($ Number of assessment forms with the data item documented/ Total number of assessment forms audited) $\times 100$

Signing and dating of assessment forms (process): a dichotomous scale was used for evaluating four measurement items: the nurse's printed full name, signature, designation and date. One point was given to the presence of each item, with a maximum four points for each form.

\section{6 Data analysis}

Raw data was entered into an Excel spreadsheet and then imported to an SPSS (1.8 version) file for statistical analysis. Descriptive statistics were used to present the results of quantity of nursing assessment documentation. As the data set was not normally distributed, the non-parametric Mann-Whitney $U$ test was employed to identify any significant differences between the paper-based and electronic systems in the number of documented assessment forms, scores of quality of nursing assessment documentation and the comprehensiveness rates of nursing assessment. Pearson's chisquared test was used to test the difference in the frequencies of nursing assessment documentation between the two systems specific to each assessment domain and data item. A p-value less than 0.05 was considered statistically significant.

\section{Results}

\section{1 The practice of nursing assessment documentation in the participating aged care homes}

There were distinct differences in documentation practices at the operational level among the participating homes, in several areas: 


\section{The use of electronic documentation systems}

Two types of commercial electronic documentation systems were used by the three participating aged care organisations: Software1 was implemented in Organisation 1 and Software 2 in Organisations 2 and 3. The two systems contained assessment forms in different formats, covering a similar scope of health status and care issues for a resident as required by the accreditation standards. Generally, the structure of the electronic forms was a close replication of the previous paper-based forms.

Most participating aged care homes had changed the platform for nursing assessment documentation from paper-based to electronic at the time of data collection. However, hybrid systems (using both paper-based and electronic) were used in Homes C and D.

\section{Organisational requirements for nursing assessment documentation}

Each of the three participating aged care organisations had their own documentation requirements with regard to the number and type of assessment forms to be used and the timeframes for the documentation of admission and ongoing assessment for a resident (Table I).

Table I. The documentation protocol of nursing assessment in each organisation

\begin{tabular}{l|l|l|l}
\hline Type of requirements & $\begin{array}{l}\text { Organization 1 } \\
\text { protocol }\end{array}$ & $\begin{array}{l}\text { Organisation 2 protocol } \\
\text { (Home A and B) }\end{array}$ & $\begin{array}{l}\text { Organisation 3 } \\
\text { protocol }\end{array}$ \\
\hline $\begin{array}{l}\text { Number of admission } \\
\text { assessment forms }\end{array}$ & 10 compulsory & 18 compulsory +9 & (Home G, H, I) \\
additional if required & homes to select \\
\hline $\begin{array}{l}\text { Timeframe for the } \\
\text { admission assessment } \\
\text { forms }\end{array}$ & Within 56 days & Within 28 days & Within 35 days \\
\hline $\begin{array}{l}\text { Timeframe for the } \\
\text { completion of an }\end{array}$ & $\begin{array}{l}\text { Overall re-assessment } \\
\text { conducted annually; }\end{array}$ & $\begin{array}{l}\text { Overall re-assessment } \\
\text { annually; two monthly }\end{array}$ & Ongoing assessment \\
\hline
\end{tabular}




\begin{tabular}{l|l|l|l}
\hline ongoing assessment & ongoing assessment & ongoing assessment and & changes \\
form & $\begin{array}{l}\text { assessment when } \\
\text { changes }\end{array}$ & condition changes & \\
& & & \\
\hline
\end{tabular}

\section{Formats of paper-based and electronic assessment forms}

Each organisation used its own format of paper-based assessment forms, which could be modified over time. All paper-based assessment forms contained pre-structured items, including tick box answers to select from, yes or no options to choose, or a data field for entering free-text narrative. A few assessment forms such as manual handling assessment and mobility assessment were in a chart format with graphics to highlight. In addition, some forms such as pain and mental status assessment forms had scales into which a score was required to be given.

Although Organisations 2 and 3 used the same electronic documentation system, the formats of the assessment forms were different. In Organisation 2, the format of assessment forms was generally standardized. In Organisation 3, their format could vary in different homes or within individual homes.

\section{2 Quantity of nursing assessment documentation in paper-based and electronic} documentation systems

Fourteen out of 159 paper-based records (9\%) did not contain any resident assessment forms. In contrast, all of the 249 electronic resident records contained documented assessment forms, either admission or ongoing.

A total of 2299 paper-based and 6997 electronic assessment forms were found in the records. The mean number of documented assessment forms in each record was 14.46 (SD 8.45) in the paper-based systems and 28.10 (SD 17.52) in the electronic systems, 
indicating a significant increase in the quantity of assessment documentation in the electronic systems $(\mathrm{p}<0.001)$.

\section{3 Completeness and timeliness of nursing assessment documentation in the paper- based and electronic documentation systems}

As shown in Table II, there was no statistically significant difference in the overall completeness scores for the paper-based and electronic admission assessment forms. However, at organisation level the scores were higher for the electronic system than the paper system in Organisation 1, but lower in Organisation 2.

The overall scores for the completeness of ongoing assessment forms and timeliness of documentation of admission assessment forms were lower in the electronic than in the paper-based systems $(\mathrm{p}=0.001)$. Such a decrease in the electronic system mainly occurred in Organisation 2.

\section{4 Comprehensiveness of assessment forms in paper-based and electronic systems}

There was a statistically significant increase in the mean comprehensiveness rate of assessment documentation in the electronic systems in comparison with the paperbased system ( 0.66 vs $0.58, \mathrm{p}=0.001)$, though this did not occur in Organisation 3.

\section{5 Frequencies of documentation of nursing assessment specific to each care domain in paper-based and electronic documentation systems}

There were variations in the frequencies of nursing assessment documentation among

16 assessment domains in both paper-based and electronic systems. The patterns of the variations in the two systems were similar (Figure 1). Statistically significant differences were found between the two systems in 10 out of 16 assessment domains $(\mathrm{p}<0.05)$. In comparison with the paper-based systems, there was a large increase in 
assessment documentation in the electronic systems for residents' pain, leisure/lifestyle and cultural needs. In contrast, there was a decrease in the electronic records for nutrition/hydration, behaviour and communication/sensory loss.

Table II. Summary scores for completeness, timeliness of completion, and comprehensiveness rates of paper-based and electronic forms at overall and organisational levels.

\begin{tabular}{|c|c|c|c|c|c|}
\hline & Mean (SD) & & Median (IQ & & $\begin{array}{l}\text { P value (Mann- } \\
\text { Whitney U } \\
\text { test) }\end{array}$ \\
\hline $\begin{array}{l}\text { Instrument questions ( } \mathrm{n}= \\
\text { sample size, paper vs } \\
\text { electronic) }\end{array}$ & $\begin{array}{l}\text { Paper- } \\
\text { based }\end{array}$ & Electronic & $\begin{array}{l}\text { Paper- } \\
\text { based }\end{array}$ & Electronic & \\
\hline $\begin{array}{l}\text { Overall score for } \\
\text { completeness of admission } \\
\text { assessment forms (129 vs } \\
147 \text { ) }\end{array}$ & $\begin{array}{c}3.24 \\
(0.54)\end{array}$ & $3.27(0.42)$ & $3.30(0.60)$ & $3.20(0.60)$ & 0.913 \\
\hline Organization 1 (29 vs 31) & $3.30(0.40)$ & $3.71(0.40)$ & $3.40(0.60)$ & $3.90(0.50)$ & $<0.001$ \\
\hline Organization 2 (50 vs 68) & $3.37(0.54)$ & $3.21(0.29)$ & $3.50(0.60)$ & $3.00(0.50)$ & 0.001 \\
\hline Organization 3 (50 vs 48) & $3.09(0.59)$ & $3.08(0.40)$ & $3.00(0.20)$ & $3.10(0.40)$ & 0.513 \\
\hline $\begin{array}{l}\text { Overall score for } \\
\text { completeness of ongoing } \\
\text { assessment forms (85 vs 164) }\end{array}$ & $3.30(0.81)$ & $3.13(0.46)$ & $3.50(1.00)$ & $3.10(0.4)$ & 0.001 \\
\hline Organization 2 (43 vs 105) & $3.39(0.83)$ & $3.14(0.53)$ & $3.60(1.00)$ & $3.20(0.50)$ & 0.002 \\
\hline Organization 3 (42 vs 59) & $3.21(0.80)$ & $3.12(0.33)$ & $3.35(1.00)$ & $3.10(0.30)$ & 0.091 \\
\hline $\begin{array}{l}\text { Overall score for timeliness } \\
\text { of completion of admission } \\
\text { assessment forms (121 vs } \\
146 \text { ) }\end{array}$ & $3.16(1.40)$ & $2.93(1.10)$ & $4.00(1.00)$ & $3.00(2.00)$ & 0.001 \\
\hline Organization 1 (26 vs 31) & $3.08(1.52)$ & $3.01(0.97)$ & $4.00(1.00)$ & $3.00(1.00)$ & 0.111 \\
\hline Organization 2 (49 vs 68) & $3.27(1.27)$ & $2.94(1.06)$ & $4.00(1.00)$ & $3.00(2.00)$ & 0.006 \\
\hline Organization 3 (46 vs 47) & $3.09(1.46)$ & $2.85(1.27)$ & $4.00(1.00)$ & $3.00(2.00)$ & 0.110 \\
\hline $\begin{array}{l}\text { Overall comprehensiveness } \\
\text { rate of admission assessment } \\
\text { forms (135 vs } 145 \text { ) }\end{array}$ & $\begin{array}{c}0.58 \\
(0.19)\end{array}$ & $\begin{array}{c}0.66 \\
(0.17)\end{array}$ & $\begin{array}{c}0.56 \\
(0.31)\end{array}$ & $\begin{array}{c}0.69 \\
(0.28)\end{array}$ & $<0.001$ \\
\hline Organization 1 (27 vs 31) & $\begin{array}{c}0.76 \\
(0.08)\end{array}$ & $0.81(0)$ & $\begin{array}{c}0.75 \\
(0.06)\end{array}$ & $0.81(0)$ & $<0.001$ \\
\hline
\end{tabular}




0.56

(0.31)

(0.25)

0.138

Notes: Organisation 1 was excluded from analysis of ongoing assessment documentation because no electronic ongoing assessment forms was collected from the organisation.

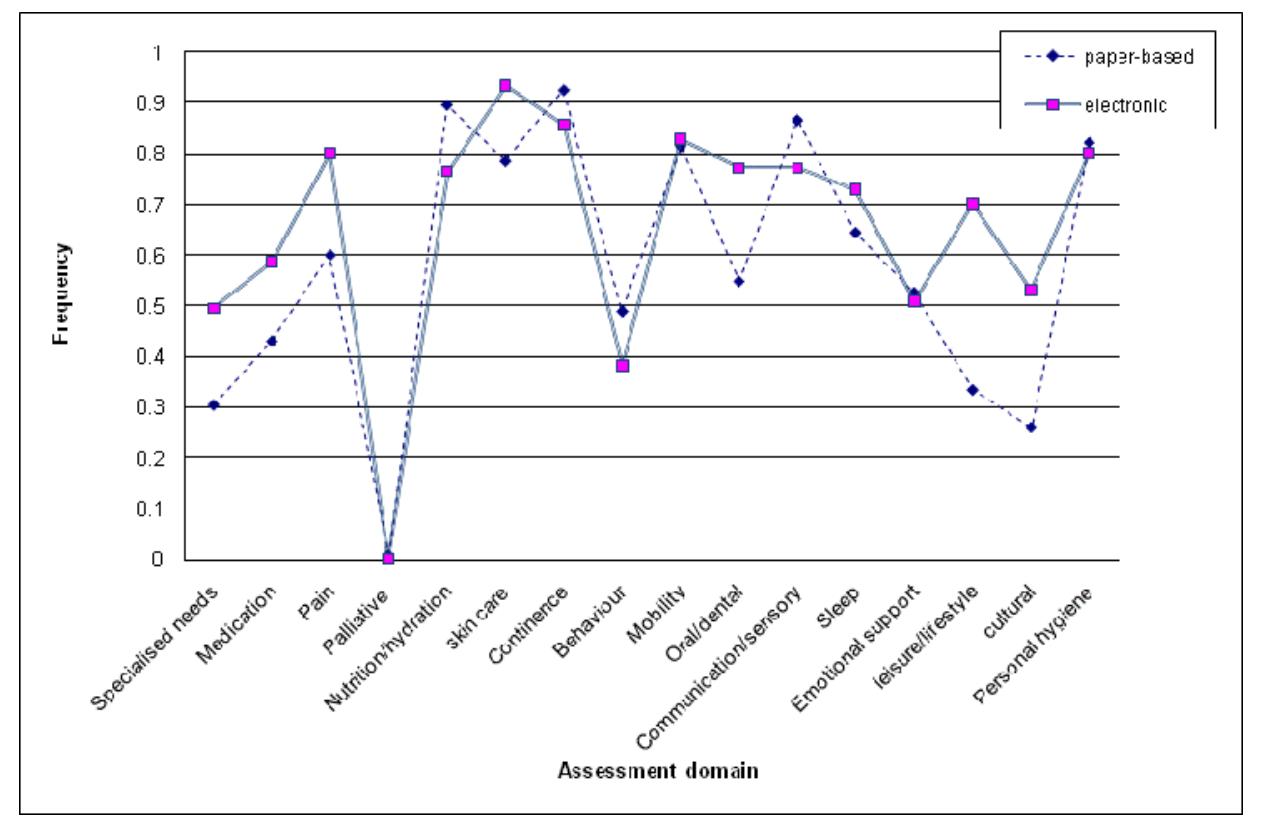

Figure 1. Frequency of assessment documentation for each required assessment domain (135 paper vs 141 electronic )

\section{6 Content, signing and dating of personal hygiene assessment forms}

In Organisation 2, three versions of paper-based forms were found. According to the dates that had been entered on the forms by nurses, these forms were used from 1999 to 2007. The number of items in the forms increased from five in the earliest version (Form 1) to 10 in a later version (Form 2) and to 51 in the latest version (Form 3). The electronic version of forms (e-Form) was an automation of Form 3, containing 26 items either derived or merged from the 51 items in Form 3. 
Most items were to collect data about a resident's functional level for carrying out particular personal hygiene tasks (e.g., washing/drying legs and back). Some items were to collect relevant data about resident preference (e.g., shower time or preferred clothing). A few items were to collect other relevant data (e.g., reason for inability to perform tasks independently and additional comments).

The level of granularity of items changed from general to specific. For example, Form 1 only had one item addressing bathing/showering in general. Four items were included in Form 2 to collect more specific relevant data (e.g., frequency of showering, use of soap). In Form 3, 25 items were designed to collect very detailed information about bathing/showering (e.g., initiate shower, collect toiletries, turn on taps/adjust temperature). In the electronic version, the 26 items cover most concepts contained in the 51 items of Form 3. The reduction of items was achieved through combining different items.

Data to be collected by the items were mostly predefined as tick-box answers in the paper-based forms and as drop-down list answers in the electronic forms. Only a few items in both paper-based and electronic forms required nurses to enter free-text data. Answers to items about resident functional levels were on an independencydependency continuum (e.g., 'independent but needing prompting', 'some assistance’ and 'full assistance').

Comparing the documentation of 26 items in e-Form $(n=70)$ with that in Form 3 $(n=19)$, documentation frequency of each item among the samples was high in both types of forms (Table III). Significant differences were found with only three items. Also there were three items that were infrequently documented in the two types of forms (frequency $<50 \%$ ). 
The mean score for signing and dating of the forms was 3.53 for the paper-based Form 3, but 4 for e-Forms $(\mathrm{P}<0.01)$.

Table III. Frequencies of documentation of items between paper-based and electronic personal hygiene assessment forms

\begin{tabular}{|c|c|c|}
\hline Items & $\begin{array}{c}\text { Form } 3 \\
(n=19)\end{array}$ & $\begin{array}{c}\text { e-Form } \\
(n=70)\end{array}$ \\
\hline Bathing & 100 & 99 \\
\hline Other & 26 & 14 \\
\hline Bathing time & 95 & 97 \\
\hline Hair care & 95 & 100 \\
\hline $\begin{array}{l}\text { Assistance required to initiate } \\
\text { shower }\end{array}$ & 100 & 100 \\
\hline $\begin{array}{l}\text { Assistance required to collect } \\
\text { toiletries }\end{array}$ & 95 & 100 \\
\hline $\begin{array}{l}\text { Assistance required to turn on } \\
\text { adjust taps }\end{array}$ & 100 & 100 \\
\hline Assistance with undressing & 100 & 100 \\
\hline $\begin{array}{l}\text { Assistance required to start } \\
\text { washing }\end{array}$ & 100 & 100 \\
\hline $\begin{array}{l}\text { Assistance required to get in } \\
\text { and out of shower }\end{array}$ & 100 & 100 \\
\hline $\begin{array}{l}\text { Assistance required to } \\
\text { wash/dry } \\
\text { hair/face/chest/groin }\end{array}$ & 100 & 100 \\
\hline $\begin{array}{l}\text { Assistance required to } \\
\text { wash/dry legs and back }\end{array}$ & 100 & 100 \\
\hline $\begin{array}{l}\text { Assistance required to get } \\
\text { dressed }\end{array}$ & 100 & 100 \\
\hline $\begin{array}{l}\text { Assistance required to choose } \\
\text { clothing }\end{array}$ & 100 & 100 \\
\hline $\begin{array}{l}\text { Assistance required to do up } \\
\text { buttons and zips }\end{array}$ & 100 & 100 \\
\hline $\begin{array}{l}\text { Assistance required to put on } \\
\text { shoes }\end{array}$ & 100 & 100 \\
\hline Assistance required to do hair & 100 & 100 \\
\hline $\begin{array}{l}\text { Assistance needed to } \\
\text { shave/apply make up }\end{array}$ & $84 *$ & $100 *$ \\
\hline $\begin{array}{l}\text { Assistance needed to with } \\
\text { nail care }\end{array}$ & 95 & 100 \\
\hline $\begin{array}{l}\text { Assistance required with } \\
\text { support stockings }\end{array}$ & $74 *$ & $100 *$ \\
\hline Assistive devices required & 95 & 91 \\
\hline $\begin{array}{l}\text { Reason for inability to } \\
\text { perform tasks independently }\end{array}$ & 100 & 84 \\
\hline Nail care & 100 & 94 \\
\hline Preferred clothing & 100 & 86 \\
\hline Prosthesis care & $47 *$ & 9* \\
\hline Other comments & 42 & 40 \\
\hline
\end{tabular}


Note: ${ }^{*}=$ statistically significant difference

\section{Discussion}

To our knowledge, this is the first study to assess the quality of nursing assessment documentation in an Australian aged care setting. It used multiple measures to address the three quality attributes of nursing assessment documentation: structure and format, process and content. This provided an overall profile about the capacity of paperbased and electronic documentation systems in aggregating resident assessment data.

\section{1 Nursing assessment documentation practice among participating aged care organizations}

There were variations in nursing assessment documentation at an operational level among the participating aged care organisations. These included differences in documentation systems, organisational documentation requirements and assessment form formats. These differences among the organisations might be determined by and again reflected the complex contextual factors [14]. For example, Organisation 3 is

the largest organisation, with a decentralized management systems. Its documentation protocol listed more than 50 assessment forms for different homes to select, so that their format was not unified across all the homes. However, Organisations 1 and 2 are smaller in size, with centralized management systems. The documentation practice in each of those organisations was standardized.

\section{2 Quantity, completeness and timeliness of nursing assessment documentation in paper-based and electronic documentation systems}

The higher quantity of assessment documentation in the electronic systems reflects better record keeping using the systems, which would support the planning and provision of individualized care to residents. 
The study results did not lend support to the electronic systems improving the completeness of nursing assessment documentation and timeliness. The mean scores for the two measures were above three out of four in both paper-based and electronic documentation systems, indicating a sound completion of the assessment documentation in either system. However, non-completion of up to one third of items and delay in documentation in either type of assessment form might impair nurses’ ability to make correct and timely judgment about a resident's needs, which could be detrimental to the planning of appropriate care.

Use of assessment forms might be affected by the platform of documentation such as the format and terminologies of the forms. Workload, timing issues, computer literacy level and organisational training strategies all might affect nurses' use of computers for electronic documentation $[15,16]$. Other factors might be the applicability of an assessment item to a resident's condition, the resident's capacity to convey valid information to a nurse during the assessment process, the nurse's attitude toward documentation, and knowledge and skill in nursing assessment and its documentation $[17,18,19]$.

\section{3 Comprehensiveness and frequency of nursing assessment documentation}

Despite differences in assessment protocols and assessment form formats among the organisations, comprehensiveness of assessment documentation was higher in the electronic documentation systems (66\%) than in the paper-based ones (58\%). This should provide better support for nurses to plan for meeting extensive resident care needs. This improvement might be attributed to the defined structure and improved access to assessment forms in the electronic systems. However, the scope of care was 
still not fully covered by the electronic forms, suggesting a need for further improvement.

The similar patterns of documentation frequencies of assessment specific to care domains in the two systems may be determined by underlying characteristics of the residents' health status and care needs, which should not be affected by the implementation of the electronic system. It might also reflect that the electronic systems did little to change nurses' documentation practice in terms of prioritising specific care needs. The decrease in electronic assessment documentation for the residents' care needs of nutrition/hydration, behaviour and communication/sensory loss suggested a need for improvement in documenting these items in the electronic systems.

\section{4 Quality of the process and content of personal hygiene documentation}

Three versions of the paper-based forms were found for hygiene assessment, with an increasing attention to resident individualised needs and personalised hygiene care over the years. The high level of granularity of the assessment items was maintained in the electronic documentation system.

Similar high rate of completion of items was found for both paper-based and electronic personal hygiene assessment forms. Two of the three items that were infrequently completed required entry of free-text comments and the other was about prosthesis care. The former might be caused by a lack of additional information or a reluctance of nurses to document additional data, whether in paper-based or electronic medium. The later might be due to inapplicability of the item on prosthesis care to most residents. 
There were four items regarding signing and dating of the assessment forms: the signature, printed name and designation of the nurse and the date when the form was filled in. These reflect the quality of documentation process which determines the reliability of resident data collected in the forms. The average score for these four measurement items was 4 for the e-Forms, but was 3.52 for the paper-based Form 3 ( $<<0.01$ ), indicating that the electronic system had facilitated the improvement of this process quality of documentation through its automatic function.

\section{5 Limitations of the study}

A convenience sampling method was used to collect data, so the sample records may not be entirely representative of the population data in the participating aged care organisations. In addition, the descriptive nature of the study does not demonstrate a conclusive causal relationship between the electronic systems and the quality of nursing assessment documentation. Factors which might have affected the findings could not be controlled in this in-situ study conducted in a natural aged care setting. For example, a new governmental aged care funding instrument (ACFI) was introduced during the period of implementation of the electronic systems in the aged care organisations [19]. Also hybrid systems were used in the two homes. These might have influenced the number of assessment forms in the electronic systems.

\section{Conclusion}

Under the same legislative requirements, there can be variation in practice of nursing assessment documentation among different aged care organisations or homes. Such varying practices may not necessarily be standardized by the use of an electronic nursing documentation system. Electronic systems could improve the quality of documentation structure and format, process and content for some components of 
nursing assessment. Although the electronic systems contained more documented assessment forms, which covered a wider range of resident care needs, they did not perform better than paper-based systems in the completeness and timeliness of documentation. Therefore, further work on the usage of the electronic systems may focus on improving these aspects. There is also a need for improvement in compliance with standards in order to address the entire care needs of residents.

Several areas may need to be further studied. The relationship between the characteristics of an item in a form and its completion status may need to be further investigated for the improvement of design or upgrade of assessment forms in electronic systems. The quality of nursing assessment documentation for focused care issues such as pain, pressure ulcer and continence needs to be understood from the clinical point of view. Such a study should provide evidence reflecting the appropriateness of items designed in a form for a specific care issue and nurses' knowledge and skills in collecting relevant clinical data. It would be also valuable to investigate the factors causing flaws in the nursing assessment documentation such as incomplete and delayed documentation. In addition, variation in nursing assessment documentation practice among the organisations and its impact on quality of care and resident outcomes need to be investigated in the future studies.

\section{Acknowledgements}

The study is a part of a broad research project funded by the Australian Research

Council Industry Linkage Grant Scheme LP0882430 and five Australian aged care organisations: Aged Care and Community Services Australia, Illawarra Retirement Trust, RSL Care, UnitingCare Ageing NSW.ACT and Warrigal Care. These organisations supported the researchers in data collection, but were not involved in 
the study design, analysis, interpretation of data and writing of the manuscript. The authors thank the residents in the participating aged care homes and their representatives for giving consent for collection and analysis of their records. The authors would also like to acknowledge nurses, nursing managers and management groups of the participant aged care organisations for giving the research team support and access to the aged care facilities to collect empirical data.

\section{Statement on conflicts of interest}

The authors claim that there is no conflict of interest with conduction of the study.

\section{Summary Table}

\begin{tabular}{|l|l|l|}
\hline What was known & Documentation of nursing assessment and its quality \\
before this study & are understudied. \\
- & Measurement of quality of nursing assessment \\
& documentation was confined to the presence of \\
& assessment data at different levels. \\
- & Electronic documentation systems have the potential \\
to improve the quality of nursing documentation. & Nursing assessment documentation practice in \\
khat this study & Australian aged care is generally defined by \\
knowledge' & legislation, but varies across organisations and aged \\
care homes. & The quality of nursing assessment documentation \\
\hline
\end{tabular}




\begin{tabular}{|c|c|}
\hline domains: format and structure, process and content. \\
The study confirms that electronic documentation \\
systems can improve the quality of nursing \\
assessment documentation in the three domains \\
measured by the metrics of quantity, \\
comprehensiveness, frequency and signing and \\
dating of documentation. \\
Electronic systems did not improve the completeness \\
and timeliness of nursing assessment documentation.
\end{tabular}




\section{References:}

[1] T.F. Kelley, D.H. Brandon, S.L. Docherty, Electronic nursing documentation as a strategy to improve quality of patient care, J Nurs Scholarship 43(2) (2011) 154-162.

[2] E. Ammenwerth, U. Kutscha, A. Kutscha, C Mahler, R. Eichstädter, R. Haux, Nursing process documentation systems in clinical routine-prerequisites and experiences, Int J Med Inform 64(2) (2001)187-200.

[3] E. Ammenwerth, F. Rauchegger, F. Ehlerset, al. Effect of a nursing information system on the quality of information processing in nursing: An evaluation study using the HIS-monitor instrument, Int J Med Inform 80(1) (2011) 25-38.

[4] M.B. Michel-Verkerke, Information Quality of a Nursing Information System depends on the nurses: A combined quantitative and qualitative evaluation, Int J Med Inform 81(10): (2012) 662- 673.

[5] H.E. Resnick, B.B. Manard, R.I. Stone, M. Alwan, Use of electronic information systems in nursing homes: United States, 2004. J Am Med Inform Assoc 16(2) (2004) 179-186..

[6] J. Crisp, C. Taylor, P.A. Potter, A.G. Perry, POTTER and PERRY'S fundamentals of nursing (2nd ed), Elsevier Australia, 2005.

[7] L. White, Documentation and the Nursing Process. Delmar Learning, Clifton Park, NY, 2002.

[8] Australian Government Department of Health and Ageing Improving the Quality of Residential Care: Residential Care Standards and Accreditation, 2007. Available from: http://www.health.gov.au/internet/main/publishing.nsf/content/ageing-rescarestandard.htm.

[9] C. Björvell, R. Wredling, I. Thorell-Ekstrand, Long-term increase in quality of nursing documentation: effects of a comprehensive intervention, Scand J Caring Sci 16 (1) (2002) 34-42.

[10] M.R., Darmer, L. Ankersen, B.G. Nielsen, G. Landberger, E. Lippert, I. Egerod, Nursing documentation audit - the effect of a VIPS implementation programme in Denmark, J Clin Nurs 15(5) (2006) 525-534.

[11] C.R. Barry, K. Brown, D. Esker, M.D. Denning, L. Kruse, E. Binder, Nursing assessment of ill nursing home residents, J Gerontol Nurs 28(5) (2002) 4-7.

[12] A. Ehrenberg, M. Ehnfors, I. Ekman, Older patients with chronic heart failure within Swedish community health care: a record review of nursing assessments and interventions, J Clin Nurs 13(1) (2004) 90-96.

[13] N. Wang, D. Hailey, P. Yu Quality of nursing documentation and approaches to its evaluation: a mixed-method systematic review, J Adv Nurs 67(9) (2011) 18581875.

[14] C. Oroviogoicoechea, B. Elliott, R. Watson, Evaluating information systems in nursing, J Clin Nurs 17 (2008) 567-575.

[15] P. Yu, D. Hailey, H. Li, Caregivers' acceptance of electronic documentation in nursing homes, J Telemed Telecare 14(5) (2008) 261-265. 
[16] P. Yu, N. Comensoli, An exploration of the barriers to the adoption of information technology in Australia aged care industry, Health Informatics Conference Brunswick East, Vic, Australia, HIC 2004.

[17] A. Cheevakasemsook, Y. Chapman, K. Francis, C. Davies, The study of nursing documentation complexities, Int J Nurs Pract 12(6) (2006) 366-374.

[18] R. Daskein, W. Moyle, D. Creedy, Aged-care nurses' knowledge of nursing documentation: an Australian perspective, J Clin Nurs 18(14) (2009) 2087-2095.

[18] T. Wibe, E. Edwin, E.H. Husby, T. Vedal, Implementation of nursing care plan in the Electronic Patient Record (EPR) findings and experiences, Stud Health Technol Inform 122 (2006) 309-313.

[19] Australian Government Department of Health and Ageing, Aged Care Funding Instrument (ACFI) User Guide, 2009. Available from:

http://www.health.gov.au/internet/main/publishing.nsf/Content/D3248F580AA9EE41 CA25738000084437/\$File/ACFI\%20User\%20Guide.pdf. 